\title{
Analysis of Multipolar Linear Paul Traps for Ion-Atom Ultracold Collision Experiments
}

\author{
M. Niranjan *, Anand Prakash and S. A. Rangwala * \\ Raman Research Institute, C. V. Raman Avenue, Sadashivanagar, Bangalore 560080, India; prakash@rri.res.in \\ * Correspondence: niranjan@rri.res.in (M.N.); sarangwala@rri.res.in (S.A.R.)
}

Citation: Niranjan, M.; Prakash, A.; Rangwala, S.A. Analysis of Multipolar Linear Paul Traps for Ion-Atom Ultracold Collision Experiments. Atoms 2021, 9, 38. https: / / doi.org/10.3390/ atoms 9030038

Academic Editors: Winthrop W. Smith and Douglas S. Goodman

\section{Received: 30 April 2021}

Accepted: 21 June 2021

Published: 29 June 2021

Publisher's Note: MDPI stays neutral with regard to jurisdictional claims in published maps and institutional affiliations.

Copyright: (c) 2021 by the authors. Licensee MDPI, Basel, Switzerland. This article is an open access article distributed under the terms and conditions of the Creative Commons Attribution (CC BY) license (https:/ / creativecommons.org/licenses/by/ $4.0 /)$.

\begin{abstract}
We evaluate the performance of multipole, linear Paul traps for the purpose of studying cold ion-atom collisions. A combination of numerical simulations and analysis based on the virial theorem is used to draw conclusions on the differences that result, by considering the trapping details of several multipole trap types. Starting with an analysis of how a low energy collision takes place between a fully compensated, ultracold trapped ion and an stationary atom, we show that a higher order multipole trap is, in principle, advantageous in terms of collisional heating. The virial analysis of multipole traps then follows, along with the computation of trapped ion trajectories in the quadrupole, hexapole, octopole and do-decapole radio frequency traps. A detailed analysis of the motion of trapped ions as a function of the amplitude, phase and stability of the ion's motion is used to evaluate the experimental prospects for such traps. The present analysis has the virtue of providing definitive answers for the merits of the various configurations, using first principles.
\end{abstract}

Keywords: ion trapping; ion-atom collisions; linear multipole traps; virial theorem

\section{Introduction}

Linear multipole Paul trap configurations are emerging as a natural choice for a wide range of charged particle trapping experiments [1-13]. The study of mixtures of trapped ions and atoms has spawned a variety of hybrid traps [3,7,14-22]. Such traps allow for the simultaneous and overlapped trapping of cold atoms and cold ions. The objective of such experiments is to study the interactions between the trapped ions and atoms, typically by collisions. It is therefore necessary to evaluate which trap geometry is ideal for experimental objectives to be met. The key question of interest here is whether higher order multi-pole traps (Figure 1) are more favorable than a quadrupole trap or not, if the objective is to study the ion-atom collisions at the coldest temperatures. The wider scope of such traps ranges from single ion-based mass spectroscopy to optical spectroscopy experiments [23-27] and a few ion-based quantum logic and computation experiments [28,29], experiments with coulomb crystals with many ions $[9,10,30-33]$ and single ions or ion clouds interacting with cold atoms [7,10,13-15,34-37].

Optical access for laser beams and absence of strong magnetic fields [34] make linear Paul traps a favorable choice for these experiments, apart from their relative simplicity of modeling and the ability to perform numerical and analytical calculations. So while the linear Paul trap can be used for a large number of experiments with different and ever expanding objectives, here we examine and evaluate different linear Paul trap electrode configurations, in an effort to decide which multipole rf linear electrode configuration is suitable for the study of ion-atom collisions at the coldest temperatures. One of the outstanding goals for hybrid-trap experiments is the realization of the s-wave regime for the ion-atom system, when there is a single trapped ion in a cloud of ultracold atoms [11,38,39].

In a Paul trap, the dynamic trapping of an ion due to the radio frequency (rf) fields applied to the electrodes results in ion motion which can be decomposed into two parts. The ion exhibits a forced motion, which is the instantaneous response of the ion to the time varying rf field (micromotion) and the slower (macro) secular motion, which is the 
trajectory of the trapped ion in the effective trapping field the ion experiences. In this scenario, the lowest energy of a single ion in a quadrupole ion trap can be confined within a compensated ion trap. Such an ion is insulated from micromotion due to the rf fields. Since a linear quadrupole ion trap has a nodal line, where the acceleration on the ion vanishes, any small deviation of the ion from this spatial location results in an increase in its motional energy. If we now consider such a specially prepared ultracold ion in collision with an ultracold atom in its vicinity, the mutual interaction between these two is sufficient to pull the ion out of the compensated configuration. The resulting collision increases the kinetic energy of the ion-atom pair compared to the s-wave limit, due to the energy coupled from the trapping field via the mutual interaction $[3,8,37]$.

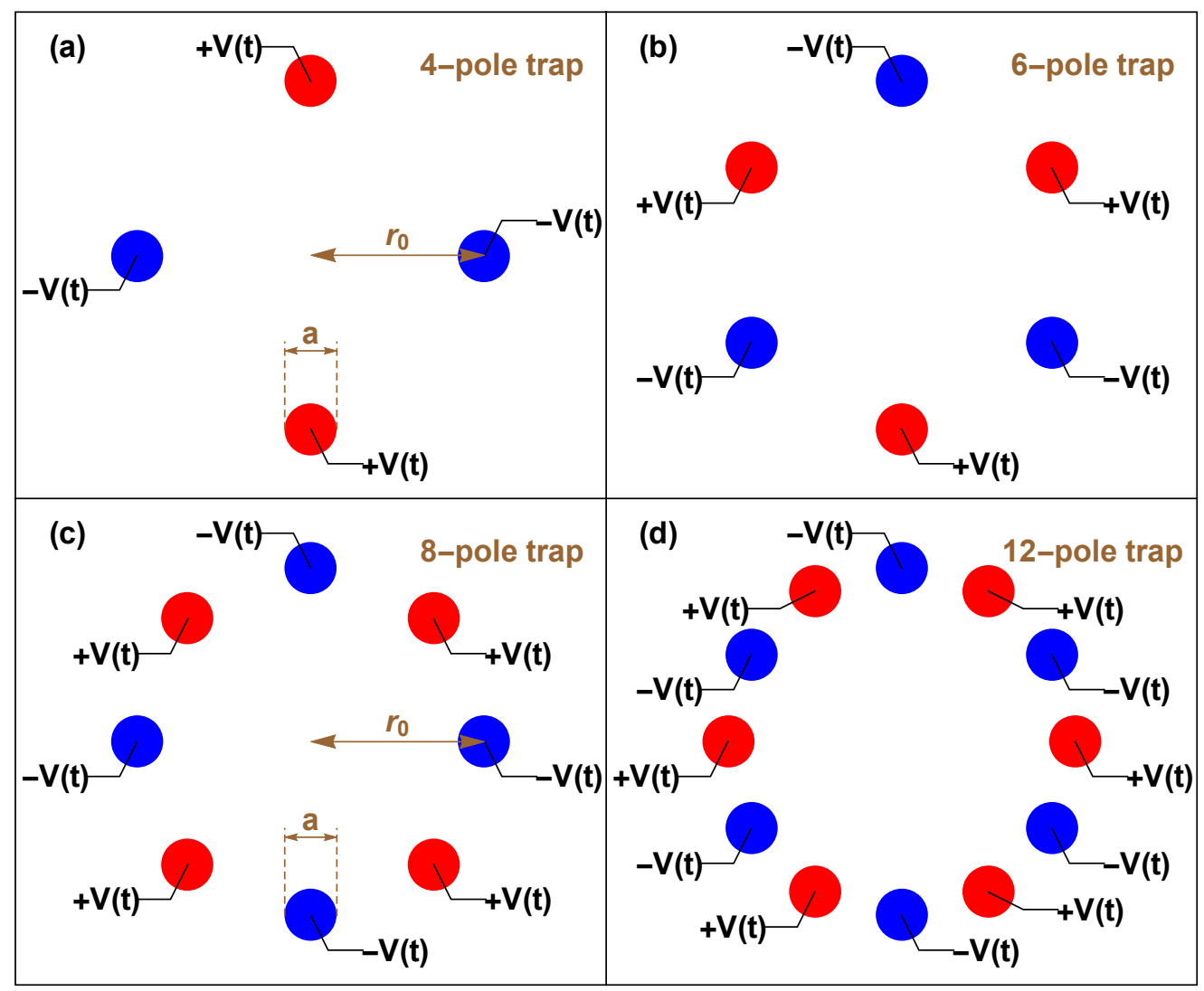

Figure 1. Electrode potential configuration for normal operation of (a) a 4-pole trap, (b) a 6-pole trap, (c) an 8-pole trap and (d) a 12-pole trap, where $V(t)=V_{0} \sin \omega t$. We keep the parameters $r_{0}$ and $a$ fixed for all the trap configurations. Note that the 12-pole trap in (d) is also equivalent to the superposition of three quadrupole traps $\left(e_{1}, e_{4}, e_{7}, e_{10}\right),\left(e_{2}, e_{5}, e_{8}, e_{11}\right)$ and $\left(e_{3}, e_{6}, e_{9}, e_{12}\right)$.

\section{Microscopic Detail of Ultracold Ion-Atom Collision}

For specificity, let us compare the scenarios of a single zero energy ${ }^{40} \mathrm{Ca}^{+}$ion at the center of a quadruple and an octupole trap colliding with a zero energy ${ }^{40} \mathrm{Ca}$ atom placed proximate to the ion. In this scenario, the mutual ion-atom interaction potential results in ion displacement from the compensated point and a collision initiates. On being displaced from the center, the ion starts moving under the influence of its trapping field, and the field does work on the ion in the presence of the atom, increasing the total energy of the colliding system. To model this collision we set the time of the first collision to be equal to $t=0$, the ion-atom interaction potential is modeled by induced dipole interaction term, $-\mathrm{C}_{4} / r^{4}$, the collision is always head on and its sense reverses when ion-atom internuclear separation approaches the repulsive wall of the ion-atom potential energy curve. The ion atom calculation is two-dimensional and the trajectories of the ion-atom pair and 
the kinetic energy of the ion in the respective traps during an instance of such a collision is illustrated in Figure 2. The collision is treated classically and all quantum aspects of the collision are ignored. When the collision initiates, under the influence of the mutual ion-atom attraction, in the presence of the rf trapping field, $\mathrm{rf}$ energy is pumped into the collision partners, increasing the collision energy as the collision progresses. This is evident for only very low energy collisions, where the time taken for the collision spans several if cycles. The collision is complex enough so that there can be several sequential collisions between the ion-atom pair before they eventually separate.
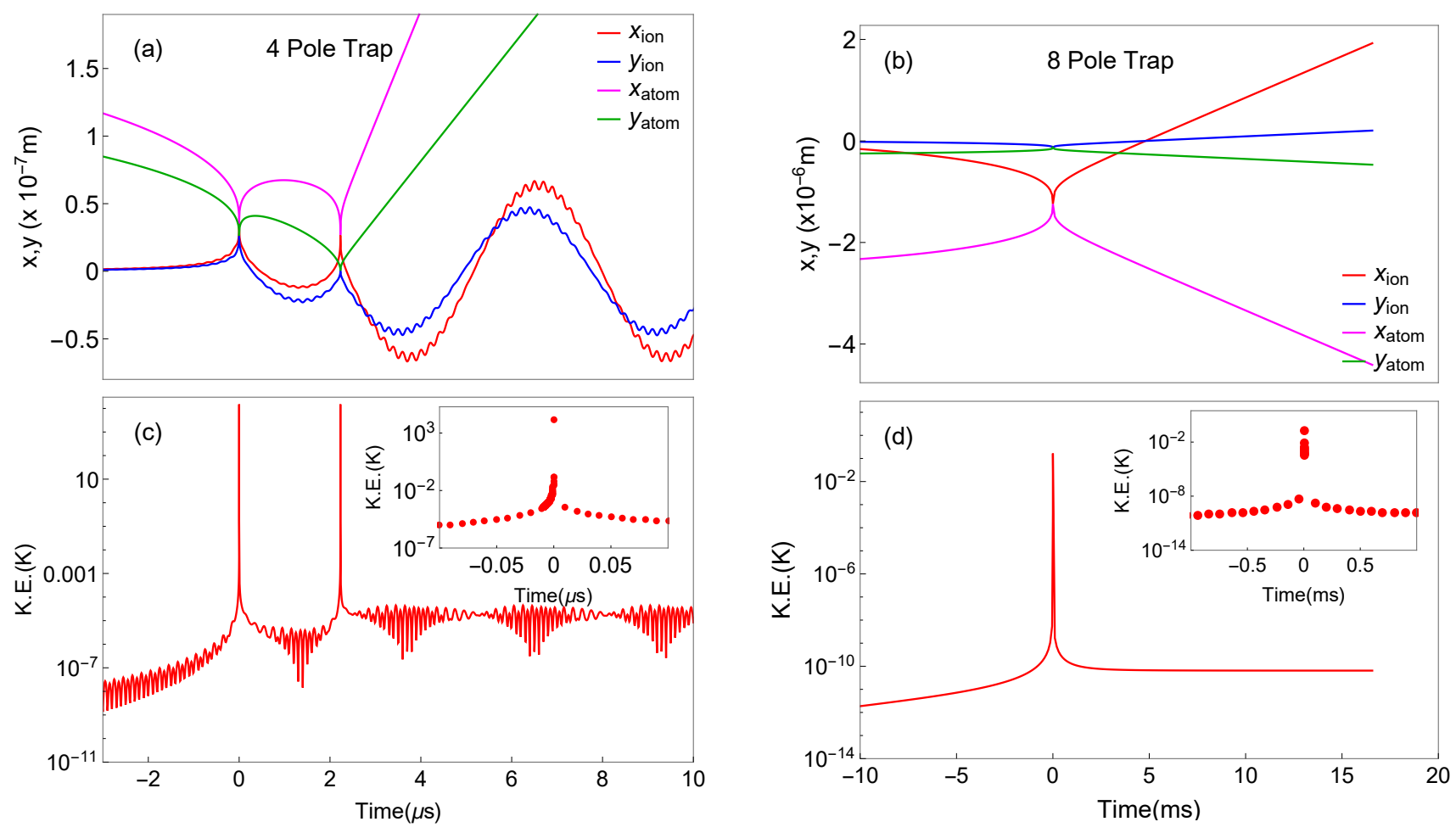

Figure 2. The time domain collision between a trapped and compensated stationary ion, initially located at the center of a quadrupole (a) and octupole (b) trap, with a stationary atom in its vicinity is shown. The calculation is two-dimensional. As a result of the mutual attraction of the trapped ion with the atom, in the presence of the trapping field, post collision, both the ion and the atom gain kinetic energy. The amount of kinetic energy gained in the collision in (a,b) is illustrated in $(\mathbf{c}, \mathrm{d})$, respectively.

An example of an ion-atom collision in an $\mathrm{rf}$ trap with quadrupole and octopole fields is shown in Figure 2. Both traps have an rf of $5 \mathrm{MHz}$, and their rf voltages are $\approx 512 \mathrm{~V}$ and $\approx 256 \mathrm{~V}$ for the quadrupole and octopole, respectively. This ensures that the Mathieu $q_{r}$ parameter is matched for the two traps, which is a fair condition to compare different traps for a compensated ion. The collision parameters are adjusted so that the first collision takes place at time $t=0$ and because of the mutual interaction of the ion and the atom before the collision, the ion shifts from its compensated position and the resulting post collision energy of the ion increases. In the process of the collision, the action of the electric field on the colliding partners has made the collision more energetic than the initial ion and atom energies would suggest. The numerical treatment for this calculation closely follows that of Cetina et al. [8].

Conventionally, most experimental efforts to study cold ion-atom collisions have favored the quadrupole trap [19], barring a few exceptions [40-43]. In contrast, for rf traps with larger numbers of electrodes, six, eight and twelve, the gradient of electric field in the neighborhood of the trap center is much smaller than that of the quadrupole. Due to this, as shown in Figure 3a similar ion-atom collision in an octopole trap would transfer significantly less energy to the ion-atom system than in the case of the quadrupole. 
Although the two traps used in Figure 2 have the same value of Mathieu parameter $q_{r}$, the secular frequencies in eight-pole trap are much smaller than that of four-pole trap. Due to the reduced rf amplitude close to the trap center, as the order of the poles increase, the energy gained by the ion during collision reduces. It should be kept in mind that the octupole trap does not have a strong restoring force at its center, resulting in the drift of the ion to regions of higher fields post collision, where the ion's micro-motion will come into play. The above example illustrates that the higher multipole trap excites less collision induced heating in a single collision event based on just the collision energy change in Figure 2, and therefore working with a higher order multipole trap appears to be advantageous. Thus, Figures 2 and 3 together provide a quantitative description of ion heating in a collision which has a direct dependence on the form of the external potential in which they collide. The figures summarize the consequences of the low energy classical ion-atom scattering problem in the presence of a time-dependent field, which has a dramatic impact on the fate of the collision. While the reduced one-dimensional problem has been solved by Cetina et al. [8] for the quadrupole potential, this two dimensional treatment is important, because of the $x^{m} y^{n}$ product terms in the higher order multipole traps, to illustrate the advantage of the octopole over the quadrupole configuration, in a quantified manner.

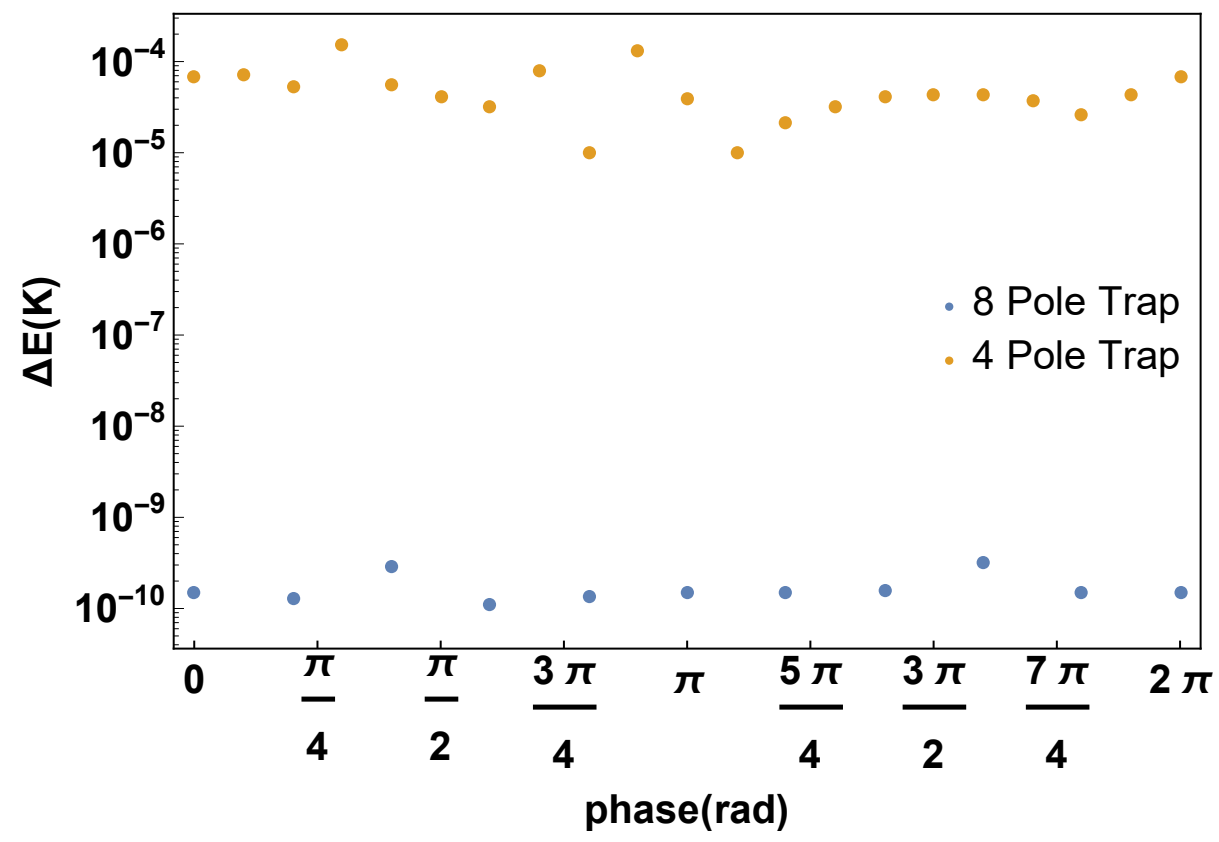

Figure 3. The change in the energy of the Ca ion-atom system, initially at rest, with the ion at the trap center post collision is presented. The results for the quadrupole and octopole trap configurations are shown. The energy gained is plotted against the phase of the electric field at the time of ion-atom's closest approach.

In the discussion that follows, we examine the totality of relevant aspects of a hybrid trap experiment and come to the conclusion that, despite the fact that the quadrupole trap transfers a significant amount of energy to the ion post collision in the ideal case, it is still the trap of choice for ultracold ion-atom experiments. There exist a number of favorable characteristics of a four-pole trap, which speak in its favor over the higher order for ultracold ion-atom experiments. We analyze in this article the other factors which are relevant to conducting the overall ion-atom hybrid trap experiment. Our analysis is part analytic, where we use the virial theorem and in part numerical. We will discuss a simple application of the virial theorem to the problem of a charged particle in the secular potential. The simulations for the trapped ion in various trap configurations illustrate the behavior of a trapped ion in the various traps and allow for detailed and nuanced analysis of the problem. 


\section{Ion Trap Configurations}

A linear multi-pole Paul trap has $2 k$ ( $k$, integer) cylindrical electrodes of diameter $a$, which are all held parallel to the trap axis $z$, such that on the transverse plane, the centers of the electrodes are equidistant points on a circle of radius $r_{0}$. Hence, such traps are referred to as $2 k$-pole traps, the simplest of which is a linear quadrupole trap ( $k=2$, 4-pole trap). In the common modes of operation, radio frequency electrical fields applied to the electrodes create a trap at the center of the geometry. This is achieved by applying ac voltages of opposite polarity to alternate electrodes, which spatially confine charged particles in the transverse direction. For confinement along the trap axis, another set of electrodes (end caps [22], segmented electrodes [29]) are used, to which dc voltages are applied in order to obtain the necessary trap depth and curvature along the symmetry axis of the $2 k$-pole trap. In what follows, we shall solve the for the ion trajectories two dimensions.

The trapped ion trajectories obey the Mathieu equation, the solution of which can be decomposed into two parts [44]. The macro-motion is characterized mainly by the pseudo-potential (proportional to $r^{2 k-2}$, where $r$ is the distance from trap center in the transverse plane) seen by the ion, which is the time averaged trapping field over the fast $\mathrm{rf}$ cycles. The micro-motion of the ion is its response to the electric field at the instantaneous position of the ion. For most experiments with ion traps, micro-motion is undesirable as it limits the cooling of the ions, induces decoherence or limits ion control.

As higher order pseudo-potentials would exhibit a potential of the kind $r^{n}, n \geq 2$ is the potential form at the center of the electrodes, ions in a higher order $2 k$-pole trap are expected to experience a lesser electric field on average compared to ions in a four-pole trap. Thus, the magnitude and effect of micro-motion would decrease with an increase in the order of a $2 k$-pole trap, which is one of the motivations for building them. This is illustrated in Figure 2, where the micromotion is not visible on the trajectories for the eight-pole trap but distinctly visible on the four-pole trap. In principle, the number of poles can be very large, though necessary $a / r_{0}$ ratios for optimum stability of the trap and geometrical constraints have seen experimental configurations up to 22 poles [45].

For each of the configurations in Figure 1, we analyze the dynamics of the ion using the virial theorem. The success and applicability of the virial approach is very valuable as, it reduces a complex dynamical non-linear problem with time variation of the potential and cross terms in the coordinates to one that can be solved almost by inspection. However, this approach needs to be validated carefully with explicit numerical trajectory calculations so the conclusions arrived at are shown to be reliable, once and for all. Since in a linear Paul trap and its extensions discussed above, the configuration comprises the multipoles and two DC biased, end cap electrodes, in the analysis for the optimal trap below, we confine ourselves to the plane perpendicular to the axis of the trap as illustrated in Figure 1. This results in simplification and still allows for the problem to be analyzed in terms of mean energy.

\section{Virial Theorem for $2 k$-Pole Traps}

Let ac voltages of $V_{0} \sin \omega t$ and $-V_{0} \sin \omega t$ be applied to alternate electrodes of a linear Paul trap, i.e., $V_{0}$ is amplitude and $\omega$ is the frequency. The 2-D pseudo potential (in the transverse plane) experienced by an ion due to the $2 k$ electrodes is given by

$$
V^{*}=\frac{k^{2} q^{2} V_{0}^{2}}{4 m \omega^{2} r_{0}^{2 k}} r^{2 k-2}
$$

where $q$ is the charge and $m$ is the mass of the ion and $r_{0}$ is the size of the trap given by the distance from the center of the trap to the center of any electrode [42]. Stable trapping of ions is achieved when the amplitude of micro-motion is smaller than the distance from 
the geometric center, i.e., when relative change in the electric field amplitude during a micro-motion cycle is small. An adiabaticity parameter $\eta$, is used to quantify this,

$$
\eta=\frac{k(k-1) 2 q V_{0}}{m \omega^{2} r_{0}^{k}} r^{k-2} .
$$

When $k=2, \eta$ is a constant (i.e., for a four-pole trap) and the well known Mathieu stability criteria apply [46]. It should be noted that the Mathieu parameter $q_{r}$ in 2-D is the same as $\eta$ and thus the four-pole ion trap is stable if $\eta \leq 0.908$. When $k>2$, stability of the ion trajectory is probabilistic and subject to initial conditions of the ion. In higher order traps, it is found that only for $\eta \geqq 0.3 \equiv \eta_{\max }$, stable ion trajectories exist. This limits the spatial extent to which the ion can span, $r_{\max }$ as $\eta$ is a function of $r($ for $k>2$ ),

$$
r_{\max }=\left[\frac{\eta_{\max } m \omega^{2} r_{0}^{k}}{2 k(k-1) q V_{0}}\right]^{1 /(k-2)}
$$

Thus, the effective trap depth for a $2 k$-pole trap is given by $V^{*}\left(r_{\max }\right)$, which is very different from that of a four-pole trap. In such a scenario, the average kinetic energy of a trapped ion is described by

$$
\left\langle T_{\text {tot }}\right\rangle=\left\langle T_{s}\right\rangle+\left\langle T_{\mu}\right\rangle,
$$

where $\left\langle T_{\text {tot }}\right\rangle$ is the total kinetic energy, $\left\langle T_{s}\right\rangle$ is the mean secular energy and $\left\langle T_{\mu}\right\rangle$ the micromotion energy. The average secular kinetic energy $\left\langle T_{s}\right\rangle$ of a particle bound by a potential of form Equation (1) is given by the virial theorem as

$$
\begin{aligned}
& \left\langle T_{s}\right\rangle=(k-1)\left\langle V^{*}\right\rangle \\
& \left\langle T_{\mu}\right\rangle=\left\langle V^{*}\right\rangle \\
& \langle U\rangle=2\left\langle V^{*}\right\rangle
\end{aligned}
$$

Therefore, the ratio between average total kinetic energy and average potential energy $\langle U\rangle$, of an ion, defined here as $v$, in a $2 k$-pole trap is

$$
v=\frac{\left\langle T_{t o t}\right\rangle}{\langle U\rangle}=k / 2 .
$$

The immediate consequence of Equation (8) is that, for the same ion energy, the larger fraction of the ion energy is kinetic. This implies that, when all other things are equal, the ion in the center of the higher order trap will be more energetic than its quadrupole counterpart, which in turn implies that the ion has to be cooled down much further in order to be centered at the trap. This is detrimental to the execution of the ion-atom collision experiment with higher order multipoles in the manner discussed in Section 2, where the ion has to be positioned at the trap center as precisely as possible in order for it to be well compensated. So while it is indeed true that there is an advantage to having an higher order trap, to keep collisional heating in check, there are problems with initializing the system, flagged by the virial theorem.

\section{Equivalence of Trajectory Calculations and Virial Results}

To quantify the results, we study numerically the dynamics of a single ion in various $2 k$-pole traps. The simulations once again were performed for a Calcium ion, ${ }^{40} \mathrm{Ca}^{+}$in two dimensions. The potentials were generated in Simion software with $r_{0}=0.707 \mathrm{~cm}$ and $a=0.1 \mathrm{~cm}$ used for the electrode sizes. The rf frequency applied, $\omega$ was kept constant for all traps at $2 \pi \times 5 \mathrm{MHz}$. 


\section{Dynamics of an Ion in a $2 k$-Pole Trap}

An electric potential $V(t)=V_{0} \operatorname{Sin}(\omega t)$ with opposite polarity was applied to alternate electrodes. The time dependent potential energy profiles for an ion, $U_{2 k}(x, y, t)$ were generated both using the ideal functional form and using SIMION [47] software and used to solve the equations of motion for the ion

$$
\begin{aligned}
& m \frac{d^{2} x}{d t^{2}}=-q \frac{\delta U_{2 k}(x, y, t)}{\delta x}, \\
& m \frac{d^{2} y}{d t^{2}}=-q \frac{\delta U_{2 k}(x, y, t)}{\delta y} .
\end{aligned}
$$

Using the trajectories obtained by solving the above coupled differential equations, we calculated the ratio of $\left\langle T_{t o t}\right\rangle$ and $\langle U\rangle$ of the ion in the trap. The values of this ratio obtained when computed for a multiple of the period of the secular motion are shown in Table 1. We show, with the numerical results, that the relation, Equation (8) derived from virial theorem holds. In the presence damping, the motion of the ion in the trap is still consistent with the virial theorem. The damping term was added to the equation of motion of the ion, to model laser cooling. In order to make the coefficient of the damping term realistic, it was calculated from the scattering force due to laser beams at saturation intensity. This calculation does not capture the stochastic nature of laser cooling and hence fails to provide a cooling limit as in the case of real laser cooled atoms. Using the trajectories of the ion calculated with and without damping, we calculated the ratio of $\left\langle T_{\text {tot }}\right\rangle$ and $\langle U\rangle$ in the trap. These numbers are given in Table 1. It can be seen that the results of the virial theorem for ion traps hold even with damping or laser cooling.

Table 1. Virial analysis of $2 k$-pole traps in normal operation. Results for the coefficient $v$ from simulated trajectories using various appropriate potentials are shown, which illustrate the compliance with Equation (8). The average values were

\begin{tabular}{|c|c|c|c|c|}
\hline & $\begin{array}{l}\text { Quadrupole Trap } \\
\qquad k=2\end{array}$ & $\begin{array}{c}\text { Hexapole Trap } \\
k=3\end{array}$ & $\begin{array}{c}\text { Octupole Trap } \\
k=4\end{array}$ & $\begin{array}{c}\text { Do-Decapole Trap } \\
\quad k=6\end{array}$ \\
\hline Ideal Potential & $\frac{V(t)\left(y^{2}-x^{2}\right)}{r_{0}^{2}}$ & $\frac{V(t)\left(3 x^{2}-y^{2}\right) y}{r_{0}^{3}}$ & $\frac{V(t)\left(6 x^{2} y^{2}-x^{4}-y^{4}\right)}{r_{0}^{4}}$ & $\frac{V(t)\left(x^{2}-y^{2}\right)\left(x^{4}-14 x^{2} y^{2}+y^{4}\right)}{r_{0}^{6}}$ \\
\hline$v$ : Theory & 1 & 1.5 & 2 & 3 \\
\hline$v:$ using ideal potential & $1.00 \pm 0.05$ & $1.50 \pm 0.07$ & $2.01 \pm 0.09$ & $3.00 \pm 0.17$ \\
\hline$v:$ using SIMION potential & $0.99 \pm 0.03$ & $1.48 \pm 0.07$ & $2.03 \pm 0.08$ & $1.01 \pm 0.20$ \\
\hline$v:$ with constant damping & $1.10 \pm 0.06$ & $1.55 \pm 0.08$ & $1.98 \pm 0.09$ & $3.04 \pm 0.19$ \\
\hline
\end{tabular}
computed over several macromotion cycles and the standard deviation is shown as the error.

The evolution of ion trajectories in the different trap potentials throws up some interesting results. In ultracold atom physics, it is normal to evolve the ion trajectories from the origin, with an initial velocity. In addition, for laser cooled ions, the ions accumulate at the bottom of the defined secular potential. When cooling multiple ions, laser cooling of ions leads to a space charge limited density distribution of the cooled ions. In quadrupole potentials, these form ion crystals [48] and in higher order multipole potentials a dense localized distribution of ions is formed, which to the best of our knowledge has not led to ion crystals being produced [43].

In the case corresponding to the single compensated ion, it is required to first produce this ion. This will be done by ionizing an atom from a distribution of atoms contained within the trap and followed by subsequent cooling of the ion. With this motivation we consider the motion of an ion within the trap volume, for the different multipolar configurations. Unlike previous studies which deal with phase space and real space trajectories in such traps for an ion created at the trap center with finite kinetic energy, we simulate trajectories for a realistic case of an ion being created away from the trap center with zero initial kinetic energy. For specific initial phase relations, the probability 
distribution of the ion position with respect to the radial coordinate is shown in Figure 4 for the multipole configurations. The insets at the top of each panel show the phase space plot of the ion trajectories in one direction. For each trajectory, the coefficient $v$ is calculated over multiple periods of the secular motion. In each case the virial theorem holds within the numerical error and its value corresponds closely to the theoretical value of the trapped ion in its secular potential $[6,49]$. For all traps, the radial position distribution for the non-zero initial phase, corresponding to the ion being confined within a lower and an upper bound in $r$. The most limiting radial confinement is seen for an ion created equidistant from the two nearest electrodes. Another observation we make is that, in this limiting case of the initial phase, the lower bound for $r$ overlaps with the initial radial position, in this case $0.1 r_{0}$, for a quadrupole trap and the upper bound for $r$ overlaps with the initial radial position for a higher order trap. The results for $k>2$ in Figure 4 show that the nature of the phase space trajectories varies significantly with the initial phase, thus implying that calculation of statistical averages is further complicated in higher order traps. This dependence on the initial phase can be attributed to the presence of $x^{m} y^{n}$ terms in the potential form of higher order traps, leading to the coupling of the motion in the two dimensions. Despite the separate nature of phase space trajectories, the virial theorem result $v=k / 2$ is found to be valid in every individual case. The real space trajectory of an ion in a four-pole trap is close to an ellipse in two dimensions and for the case of the limiting phase, the lower bound of the radial orbit occurs at $0.1 r_{0}$ and, due to micromotion, the upper bound of the radial coordinate appears as broadened in the probability densities shown in Figure $4 \mathrm{a}-\mathrm{c}$. However, the real space trajectory of an ion in traps with $k>2$ spans a circularly symmetric region between two radial bounds. The inner bound occurs at $r=0$ for $\theta=0$ and at a finite radius for non-zero $\theta$, this manifests as the two radial peaks, which are sharply cut-off, in the density distributions in Figure 4 for higher pole traps, at the turning points. The radial confinement for the limiting value of $\theta$ is narrowest for $k=3$ and becomes wider for higher $k$ traps.

To address what the chance is of finding an ion at a particular distance from the trap center, we calculated two scenarios. In the first, illustrated in Figure 5a, the probability distribution of the ion created at a distance of $0.1 r_{0}$ from the origin, with zero initial velocity and at different angular displacements with respect to the electrode axes was computed. As seen from the probability density plots in Figure 4, the ion coverage of the trap volume has tremendous variety. It is therefore important to understand the statistical likelihood of the ion occupying a particular region of space. This was therefore studied with respect to the angular creation of the ion in the multipole trap in Figure 5a and its inset. The most reasonable experimental situation is that there is an cold atom ensemble located about the ion trap center. If the ion is to be created from such an ensemble of atoms by threshold ionization, what would the spatial distribution of the ion be? This is illustrated in Figure $5 \mathrm{~b}$ where the probability distribution of the ion created at a distance generated from a two-dimensional Gaussian distribution of width $0.05 r_{0}$, with zero initial velocity is plotted. The spatial density distribution of the ion, over repeated instances, is illustrated in the inset. So, Figures 4 and 5 allow us to conclude what the trapped ion distribution would be that we would have to deal with in an experiment, in particular instances and on average. 

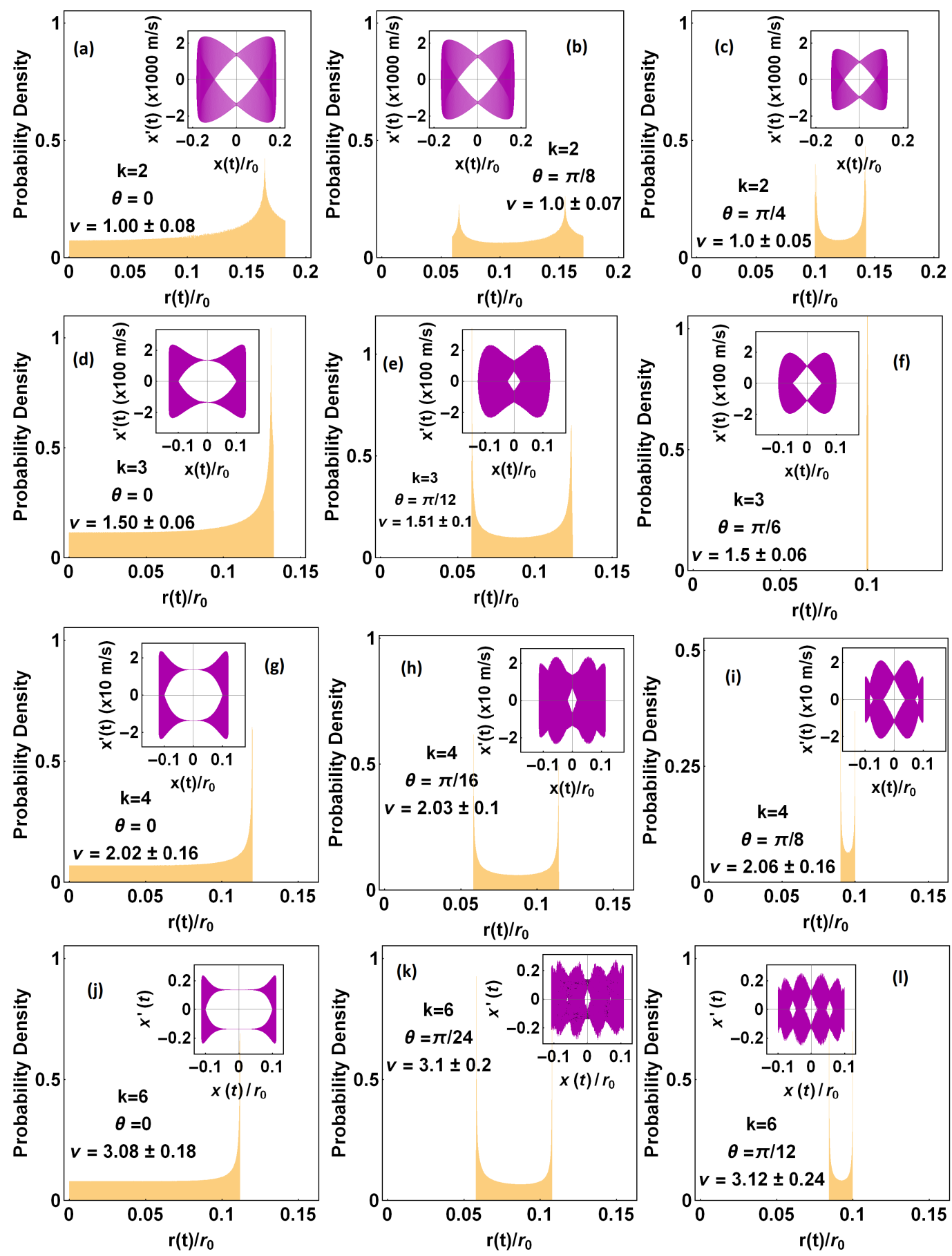

Figure 4. We show the probability distribution for the radial position coordinate, $r$, in different traps for a stationary ion which is created at $t=0$, with initial position of radius $r(0)=0.1 r_{0}$ and azhimuth angle $\theta$; we also show the corresponding phase space trajectories in the insets. Our results reveal that all higher order pole traps differ from quadrupole traps in some ways. Trap dynamics with these realistic initial conditions have not been studied earlier explicitly. The results in panels $(\mathbf{a}-\mathbf{c})$ for quadrupole trap, show that, change in $\theta$ does not effect the form of phase space density. This is in accordance with the other studies where the ion is created at $r(0)=0$ with non-zero velocities. However, our results for higher order traps in panels $(\mathbf{d}-\mathbf{l})$, unveil completely different behavior of ion motion in practical higher pole traps. Not only are the position distributions unique with characteristic sharp cut offs at $r=r(0)$, but the nature of the phase space trajectory shown in the insets, also varies significantly with $\theta$. This is important to be factored in when working with higher pole traps while evaluating ensemble average quantities. 

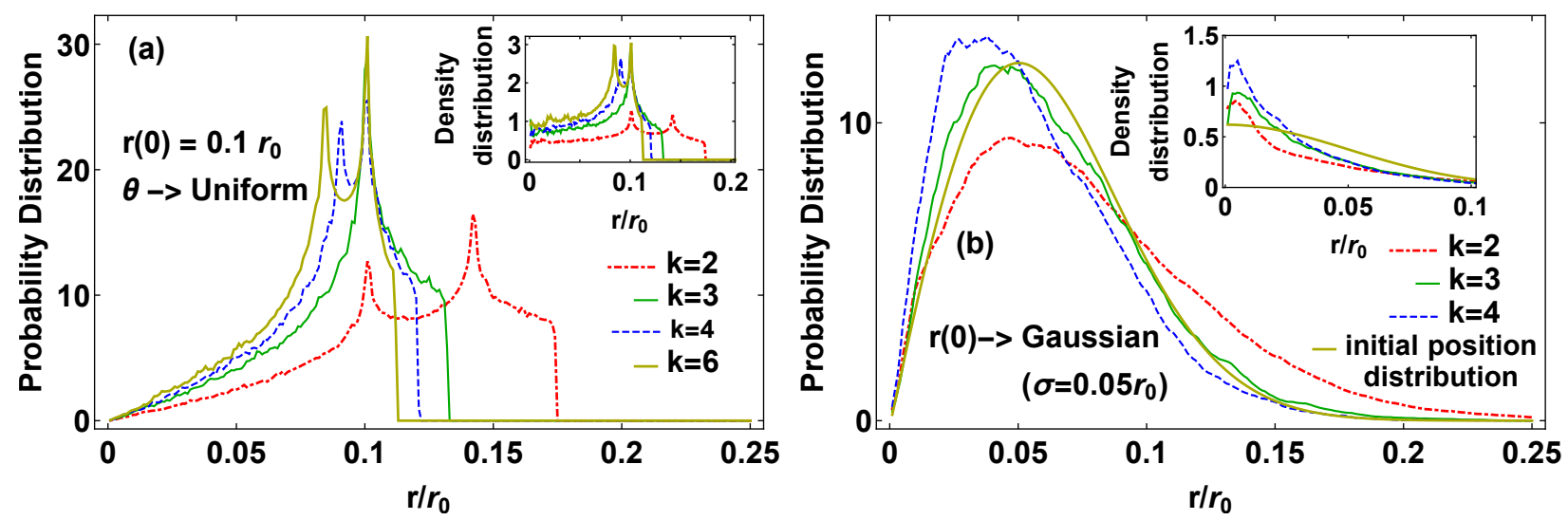

Figure 5. Panel (a) shows the probability distribution for an ion created at radial distance of $0.1 r_{0}$, with random angular coordinate $\theta$ for various $2 k$-pole traps. Panel (b) shows the probability distribution for an ion created with a Gaussian distribution of width $0.05 r_{0}$, centered with the ion trap for various $2 k$-pole traps. The insets show the corresponding density distribution.

The agreement of the hexapole and the do-decapole electrode configuration with the virial theorem is shown in table one. All values are as expected, except in the case for the do-decapole SIMION trajectory calculation, where the ratio of $\left\langle T_{t o t}\right\rangle /\langle U\rangle$ is consistent with that of a quadrupole. We presume that this is a finite grid and grid size numerical effect, where the pseudo-potential is effectively decomposed into three independent quadrupole potentials, which is what is reflected in the value of the $\left\langle T_{\text {tot }}\right\rangle /\langle U\rangle$ ratio in that instance. When the potentials analytic form is used, the value of $v=3$ results for the same ratio, as per expectations both with and without damping. It is therefore an instance where one has to be cautious with the results of explicit numerical calculations. This instance underlines the necessity for cross checking the qualitative and the explicit calculations against each other.

\section{Discussion and Conclusions}

We first observe that the agreement of the computed ion motion with the virial theorem holds well, for all the multipole traps considered here. It is therefore reasonable to conclude that an analysis based on the proper application of the virial theorem provides a legitimate platform for discussions on what to expect of ion traps, without performing detailed computations. Since we initiate the ions at a distance from the center with zero energy, we do not have a length scale for each trap corresponding to the energy of the ions; therefore, we chose to keep the parameter $q_{r}$ fixed when comparing various multipole traps. From the discussion above, given the same rf frequency and fixing the operating value of $q_{r}=0.1$ for all multipole traps, we see that with increasing $k$, the trap depths reduce. This is the consequence of the fact that for a constant $q_{r}, V_{0} \propto 1 / k$, so, as the number of poles increases, the $\mathrm{rf}$ voltage required for a stable trajectory decreases. In addition $r_{\max } \propto\left[1 / k(k-1) V_{0}\right]^{(1 /(k-2))}$, which means the radius for which the adiabatic operating condition of the ion trap persists, shrinking with increasing $k$. If the ion motion is not described by the adiabatic condition, then the conventional description of the trapped ion motion breaks down and the motion becomes sensitive to small changes. This implies that the kinetic energy of the trapped ion has to be lower at the time of production. That is determined by the extent of cooling of the atoms from which the ion is created, which cannot be reduced arbitrarily. Creating an ion with external ionization processes needs to be performed with greater care for higher order multipole traps.

Within the regime of applicability of the virial theorem, $\left\langle T_{t o t}\right\rangle /\langle U\rangle=k / 2$ applies, as seen in Table 1. When we allow for the presence of stray fields and field inhomogeneities, the requirement of a minimum trap depth is produced. In this situation, if a certain minimum trap depth is required to hold the ion, then the kinetic energy is increases as $k / 2$ 
times the mean trap potential energy. This can be problematic in the actual realization of a highly controlled multipole trap.

A practical issue for higher order multipole traps is that the secular motion slows down with respect to the applied rf frequency. It therefore takes the ion much more time to span the full phase space for the trapping conditions, and this time increases with the number of multipoles. While this poses a significant computational challenge, it is also detrimental to the time scales of typical dilute gas experiments. This is because the precision with which the ion's state can be known is harder to determine and therefore to set up a controlled ultracold ion-atom experiment of the kind described in the beginning without the deleterious effect of the rf excitation of the ion during collision becomes more difficult with larger $k$.

For the same $q_{r}$ and $r f$ frequency, higher order multi-pole ion traps have significantly lower trap depths, compared to the corresponding quadrupole trap. When $k>2$, the spatial extent of stable trapping is much smaller than the physical size of the trap, unlike in a quadrupole trap. This result, when seen in the context of a large number of ions in the trap and relative ion density in higher order traps, is at its maximum for a finite $r$, and the density at $r=0$, decreases as $2 k$ increases. This is undesirable for interactions with spatially localized cold atoms.

Another problem of higher order traps is that the pseudo-potential becomes more and more flat in a higher multi-pole trap, the cooled ion is not spatially confined to the center of the trap. This is likely to be a problem for experiments where ion localization is required. Another deduction that can be made from the virial theorem analysis is that the prospects of laser cooled ion crystals in higher order multipole traps is quite bleak. This is because, for crystals to be created, multiple points in space are required where the net force due to laser cooling, external drive fields and ion-ion repulsion become zero. Only this will allow the ions to be motionless at those locations and therefore crystallize. However the cooling requirements for the ion in higher order traps, as discussed above, is much more stringent. This is perhaps why, in higher order multipole traps, ion crystals have remained elusive, despite advances in laser cooling.

Recent studies with higher order multipole traps have shown that the flat potential is dimpled due to field imperfections and this results in a potential which has local quadrupole minimas within the higher order multipole field [43,45]. In this scenario, the lowest point of the higher order multipole trap converts into a defined number of quadrupole traps, depending on the order of the multipole. So, in the case of really efficient cooling, it is quite likely that the ions find themselves in the dimpled quadrupole potential, which is sensitive to the local field environment. However, since these local minimas are not at the geometric center of the trap, the utility of these for interactions with a localized atomic ensemble is moot. In this case, when the ion is trapped in the local quadrupole field, the discussion accompanying Figures 2 and 3 has limited applicability.

Quadrupole traps have some advantages over higher $2 k$-pole traps like larger trap depths, stability, better ion loading probability and deterministic stability. In order to capitalize on the advantages of both kinds of traps when needed, one can use either two concentric traps, one four-pole and another a higher $2 k$-pole trap or operate a higher $2 k$-pole configuration with modified polarities on different electrodes to make it an equivalent four-pole trap. Implementation of higher order linear multipole trap setup can provide versatility for experiments. In Figure 6, we show a few examples of alternate operation schemes for obtaining an equivalent quadrupole trap. This gives the opportunity to switch between a quadrupole and a higher order trap to exploit the best of both configurations. The large trap depths of the quadrupole trap and better shielding from stray electric fields can be accessed in the effective quadrupole schemes and relatively low electric field experienced by ions in higher order traps near the trap center can be accessed in the regular $2 k$-pole trap scheme, making it a versatile tool. While the configurations shown in Figure $6 b-d$ lead to an effective quasi-quadrupole trap, the scheme shown in Figure $6 a$ 
leads to a pure quadrupole trap with the additional grounded electrodes ensuring the points of zero potential, thereby providing additional stability against stray fields.

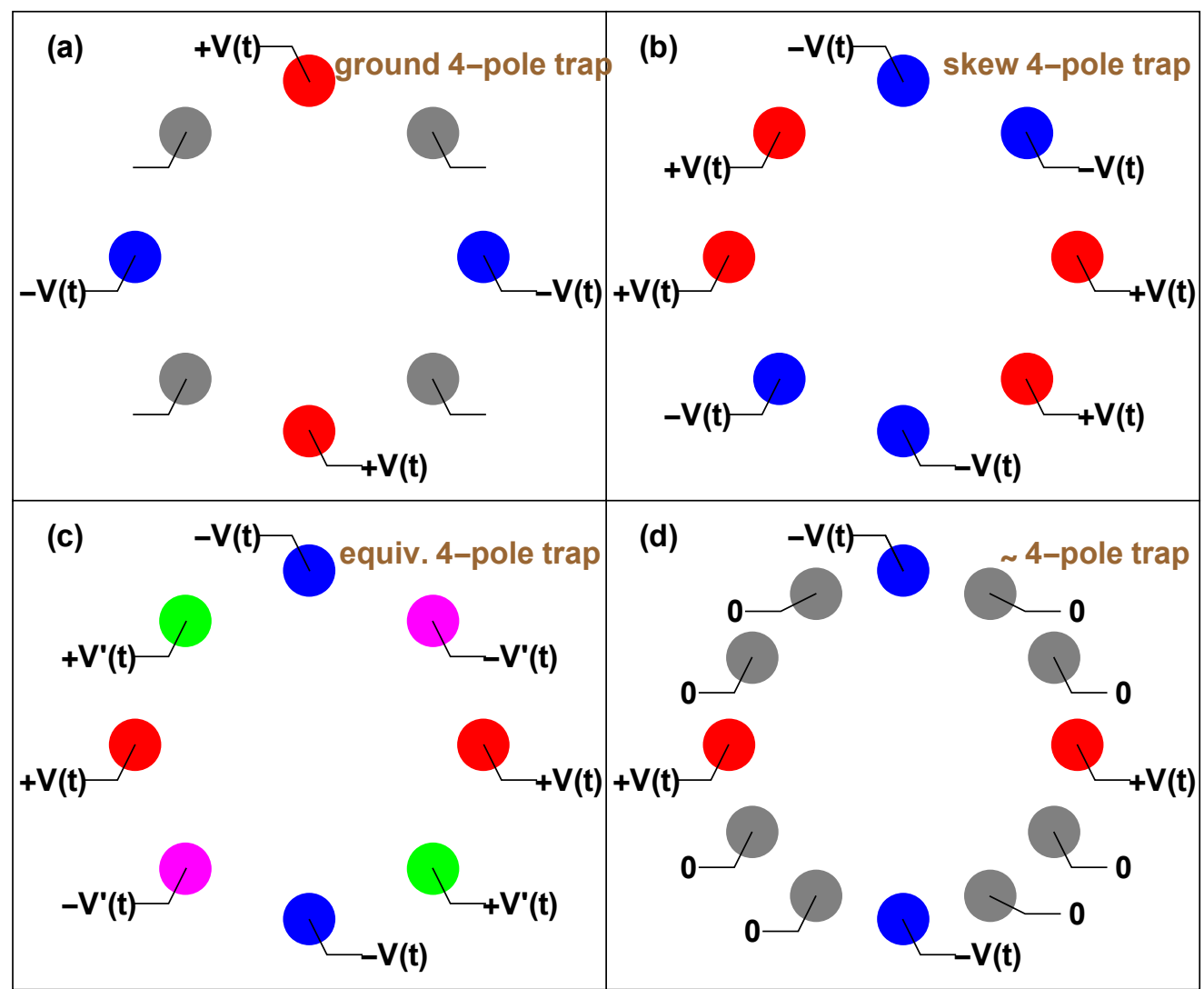

Figure 6. Alternate trap configurations for using an 8-pole or 12-pole setup as a modified quadrupole are shown. Panel (a) is an 8-pole structure with alternate electrodes explicitly grounded. Panel (b) shows a skewed quadrupole trap. Panel (c) shows an effective quadrupole trap with an 8-pole setup, where successive electrodes are at a phase difference of Pi/2. Finally, panel (d) shows a 12-pole structure, with a quadrupole field configuration and multiple shielding electrodes.

Putting together all the above results, it is not easy to see the practical advantage of using a higher order multipole trap to confine the ion for ultracold ion-atom physics. This is because such experiments are performed with both positional and state control of the colliding partners and the spread in position of the ion for a higher order multipole is experimentally hard to overcome. It is perhaps more advantageous to work with a very weak, well shielded quadrupole trap. The trap configurations in Figure 6 are a path to harness the advantages of the quadrupole trap and the higher order multipole trap. However such a trap configuration may present significant operational challenges. The virial theorem and its explicit verification provides the base for quick conclusions about energetics, which is very useful for complicated potentials. On the other hand, if the objective is to study collisions at higher energy between atoms and neutrals in buffer gas regimes then higher order multipoles can be used very fruitfully for a vast array of experiments, keeping in mind the arguments made above [40,50].

Author Contributions: M.N. and S.A.R. conceptualized the problem. M.N. and A.P. performed computations supervised by S.A.R. and all authors wrote the manuscript. All authors have read and agreed to the published version of the manuscript. 
Funding: Authors acknowledge support from Ministry of Electronics \& Information Technology (MeitY), Government of India, under grant for "Centre for Excellence in Quantum Technologies" with Ref. No. 4(7)/2020-ITEA.

Institutional Review Board Statement: Not applicable.

Informed Consent Statement: Not applicable.

Conflicts of Interest: The authors declare no conflict of interest.

\section{References}

1. Paul, W. Electromagnetic traps for charged and neutral particles. Rev. Mod. Phys. 1990, 62, 531-540. [CrossRef]

2. Major, F.G.; Gheorghe, V.N.; Werth, G. Charged Particle Traps, 1st ed.; Springer: Berlin/Heidelberg, Germany, 2005. [CrossRef]

3. Grier, A.T.; Cetina, M.; Oručević, F.; Vuletić, V. Observation of Cold Collisions between Trapped Ions and Trapped Atoms. Phys. Rev. Lett. 2009, 102, 223201. [CrossRef]

4. Zipkes, C.; Palzer, S.; Sias, C.; Köhl, M. A trapped single ion inside a Bose-Einstein condensate. Nature 2010, 464, 388-391. [CrossRef]

5. Hall, F.; Aymar, M.; Bouloufa, N.; Dulieu, O.; Willitsch, S. Light-Assisted Ion-Neutral Reactive Processes in the Cold Regime: Radiative Molecule Formation versus Charge Exchange. Phys. Rev. Lett. 2011, 107, 243202. [CrossRef] [PubMed]

6. Ravi, K.; Lee, S.; Sharma, A.; Werth, G.; Rangwala, S.A. Cooling and stabilization by collisions in a mixed ion-atom system. Nat. Commun. 2012, 3, 1126. [CrossRef] [PubMed]

7. Sivarajah, I.; Goodman, D.S.; Wells, J.E.; Narducci, F.A.; Smith, W.W. Evidence of sympathetic cooling of $\mathrm{Na}^{+}$ions by a Na magneto-optical trap in a hybrid trap. Phys. Rev. A 2012, 86, 63419. [CrossRef]

8. Cetina, M.; Grier, A.T.; Vuletić, V. Micromotion-induced limit to atom-ion sympathetic cooling in Paul traps. Phys. Rev. Lett. 2012, 109. [CrossRef]

9. Hall, F.H.J.; Eberle, P.; Hegi, G.; Raoult, M.; Aymar, M.; Dulieu, O.; Willitsch, S. Ion-neutral chemistry at ultralow energies: Dynamics of reactive collisions between laser-cooled $\mathrm{Ca}^{+}$ions and $\mathrm{Rb}$ atoms in an ion-atom hybrid trap. Mol. Phys. 2013, 111, 2020-2032. [CrossRef]

10. Härter, A.; Denschlag, J.H. Cold atom-ion experiments in hybrid traps. Contemp. Phys. 2014, 55, 33-45. [CrossRef]

11. Meir, Z.; Pinkas, M.; Sikorsky, T.; Ben-shlomi, R.; Akerman, N.; Ozeri, R. Direct Observation of Atom-Ion Nonequilibrium Sympathetic Cooling. Phys. Rev. Lett. 2018, 121, 53402. [CrossRef] [PubMed]

12. Albert, M.; Marler, J.P.; Herskind, P.F.; Dantan, A.; Drewsen, M. Collective strong coupling between ion Coulomb crystals and an optical cavity field: Theory and experiment. Phys. Rev. A 2012, 85, 023818. [CrossRef]

13. Rouse, I.; Willitsch, S. Superstatistical Energy Distributions of an Ion in an Ultracold Buffer Gas. Phys. Rev. Lett. 2017, 118, 143401. [CrossRef]

14. Schmid, S.; Härter, A.; Denschlag, J.H. Dynamics of a Cold Trapped Ion in a Bose-Einstein Condensate. Phys. Rev. Lett. 2010, 105, 133202. [CrossRef]

15. Smith, W.W.; Goodman, D.S.; Sivarajah, I.; Wells, J.E.; Banerjee, S.; Côté, R.; Michels, H.H.; Mongtomery, J.A.; Narducci, F.A. Experiments with an ion-neutral hybrid trap: Cold charge-exchange collisions. Appl. Phys. B 2014, 114, 75-80. [CrossRef]

16. Schowalter, S.J.; Dunning, A.J.; Chen, K.; Puri, P.; Schneider, C.; Hudson, E.R. Blue-sky bifurcation of ion energies and the limits of neutral-gas sympathetic cooling of trapped ions. Nat. Commun. 2016, 7, 12448. [CrossRef]

17. Ray, T.; Jyothi, S.; Ram, N.B.; Rangwala, S.A. A thin wire ion trap to study ion-atom collisions built within a Fabry-Perot cavity. Appl. Phys. B 2014, 114, 267-273. [CrossRef]

18. Jyothi, S.; Ray, T.; Ram, B.; Rangwala, S. Hybrid Ion, Atom and Light Trap; IOS Press: Amsterdam, The Netherlands, 2015; Volume 189, pp. 269-278.

19. Tomza, M.; Jachymski, K.; Gerritsma, R.; Negretti, A.; Calarco, T.; Idziaszek, Z.; Julienne, P.S. Cold hybrid ion-atom systems. Rev. Mod. Phys. 2019, 91. [CrossRef]

20. Dieterle, T.; Berngruber, M.; Hölzl, C.; Löw, R.; Jachymski, K.; Pfau, T.; Meinert, F. Transport of a Single Cold Ion Immersed in a Bose-Einstein Condensate. Phys. Rev. Lett. 2021, 126, 33401. [CrossRef] [PubMed]

21. Zipkes, C.; Ratschbacher, L.; Palzer, S.; Sias, C.; Köhl, M. Hybrid quantum systems of atoms and ions. J. Phys. Conf. Ser. 2011, 264, 12019. [CrossRef]

22. Ravi, K.; Lee, S.; Sharma, A.; Werth, G.; Rangwala, S.A. Combined ion and atom trap for low-temperature ion-atom physics. Appl. Phys. B 2012, 107, 971-981. [CrossRef]

23. Gerlich, D. Inhomogeneous RF Fields: A Versatile Tool for the Study of Processes with Slow Ions; John Wiley \& Sons, Inc.: New York, NY, USA, 1992. [CrossRef]

24. Douglas, D.J.; Frank, A.J.; Mao, D. Linear ion traps in mass spectrometry. Mass Spectrom. Rev. 2005, 24, 1-29. [CrossRef] [PubMed]

25. Asvany, O.; Brünken, S.; Kluge, L.; Schlemmer, S. COLTRAP: A 22-pole ion trapping machine for spectroscopy at 4 K. Appl. Phys. B 2014, 114, 203-211. [CrossRef] 
26. Tian, Y.; Decker, T.K.; McClellan, J.S.; Bennett, L.; Li, A.; De la Cruz, A.; Andrews, D.; Lammert, S.A.; Hawkins, A.R.; Austin, D.E. Improved Miniaturized Linear Ion Trap Mass Spectrometer Using Lithographically Patterned Plates and Tapered Ejection Slit. J. Am. Soc. Mass Spectrom. 2018, 29, 213-222. [CrossRef]

27. Schwartz, J.C.; Senko, M.W.; Syka, J.E.P. A two-dimensional quadrupole ion trap mass spectrometer. J. Am. Soc. Mass Spectrom. 2002, 13, 659-669. [CrossRef]

28. Kaplan, H.B.; Guo, L.; Tan, W.L.; De, A.; Marquardt, F.; Pagano, G.; Monroe, C. Many-Body Dephasing in a Trapped-Ion Quantum Simulator. Phys. Rev. Lett. 2020, 125, 120605. [CrossRef]

29. Ragg, S.; Decaroli, C.; Lutz, T.; Home, J.P. Segmented ion-trap fabrication using high precision stacked wafers. Rev. Sci. Instrum. 2019, 90, 103203. [CrossRef]

30. Bermudez, A.; Schaetz, T.; Plenio, M.B. Dissipation-Assisted Quantum Information Processing with Trapped Ions. Phys. Rev. Lett. 2013, 110, 110502. [CrossRef]

31. Dantan, A.; Herskind, P.; Marler, J.P.; Albert, M.; Drewsen, M. Collective strong coupling with ion Coulomb crystals in an optical cavity. In Proceedings of the CLEO/Europe-EQEC 2009-European Conference on Lasers and Electro-Optics and the European Quantum Electronics Conference, Munich, Germany, 14-19 June 2009; p. 1. [CrossRef]

32. Sterk, J.D.; Luo, L.; Manning, T.A.; Maunz, P.; Monroe, C. Photon collection from a trapped ion-cavity system. Phys. Rev. A 2012, 85, 062308. [CrossRef]

33. Ewald, N.V.; Feldker, T.; Hirzler, H.; Fürst, H.A.; Gerritsma, R. Observation of Interactions between Trapped Ions and Ultracold Rydberg Atoms. Phys. Rev. Lett. 2019, 122, 253401. [CrossRef]

34. Major, F.G.; Dehmelt, H.G. Exchange-Collision Technique for the rf Spectroscopy of Stored Ions. Phys. Rev. 1968, 170, 91-107. [CrossRef]

35. Karpa, L.; Bylinskii, A.; Gangloff, D.; Cetina, M.; Vuletić, V. Suppression of Ion Transport due to Long-Lived Subwavelength Localization by an Optical Lattice. Phys. Rev. Lett. 2013, 111, 163002. [CrossRef] [PubMed]

36. Wang, L.; Raithel, M.D.G.; Denschlag, J.H. Optical control of atom-ion collisions using a Rydberg state. J. Phys. B At. Mol. Opt. Phys. 2020, 53, 134005. [CrossRef]

37. Ben-shlomi, R.; Vexiau, R.; Meir, Z.; Sikorsky, T.; Akerman, N.; Pinkas, M.; Dulieu, O.; Ozeri, R. Direct observation of ultracold atom-ion excitation exchange. Phys. Rev. A 2020, 102, 31301. [CrossRef]

38. Schmidt, J.; Lambrecht, A.; Weckesser, P.; Debatin, M.; Karpa, L.; Schaetz, T. Optical Trapping of Ion Coulomb Crystals. Phys. Rev. X 2018, 8, 21028. [CrossRef]

39. Schmid, T.; Veit, C.; Zuber, N.; Löw, R.; Pfau, T.; Tarana, M.; Tomza, M. Rydberg Molecules for Ion-Atom Scattering in the Ultracold Regime. Phys. Rev. Lett. 2018, 120, 153401. [CrossRef]

40. Wester, R. Cold Molecular Ions in Traps. In Trapped Charged Particles; Advanced Textbooks in Physics; World Scientific (Europe): London, UK, 2015; pp. 321-334. [CrossRef]

41. Debatin, M.; Kröner, M.; Mikosch, J.; Trippel, S.; Morrison, N.; Reetz-Lamour, M.; Woias, P.; Wester, R.; Weidemüller, M. Planar multipole ion trap. Phys. Rev. A 2008, 77, 33422. [CrossRef]

42. Prestage, J.D.; Tjoelker, R.L.; Maleki, L. Mercury-ion clock based on linear multi-pole ion trap. In Proceedings of the 2000 IEEE/EIA International Frequency Control Symposium and Exhibition (Cat. No.00CH37052), Kansas City, MO, USA, 9 June 2000; pp. 706-710. [CrossRef]

43. Pedregosa-Gutierrez, J.; Champenois, C.; Houssin, M.; Kamsap, M.R.; Knoop, M. Correcting symmetry imperfections in linear multipole traps. Rev. Sci. Instrum. 2018, 89, 123101. [CrossRef]

44. Härter, A.; Krükow, A.; Brunner, A.; Hecker Denschlag, J. Long-term drifts of stray electric fields in a Paul trap. Appl. Phys. B 2014, 114, 275-281. [CrossRef]

45. Otto, R.; Hlavenka, P.; Trippel, S.; Mikosch, J.; Singer, K.; Weidemüller, M.; Wester, R. How can a 22-pole ion trap exhibit 10 local minima in the effective potential? J. Phys. B At. Mol. Opt. Phys. 2009, 42. [CrossRef]

46. Todd, J.F.J.; March, R. Practical Aspects of Trapped Ion Mass Spectrometry, Volume V: Applications of Ion Trapping Devices, 1st ed.; CRC Press: Boca Raton, FL, USA, 2009. [CrossRef]

47. Dahl, D.M. SIMION (R) 8.1 User Manual; Adaptas Solutions, LLC: Palmer, MA, USA, 2008.

48. Thompson, R.C. Ion Coulomb crystals. Contemp. Phys. 2015, 56, 63-79. [CrossRef]

49. Lee, S. The Study of Trapped Ion Collisions with Cold Atoms and Cold Molecules. Ph.D. Thesis, Raman Research Institute, Bangalore, India, 2014.

50. Mihalcea, B.M.; Giurgiu, L.C.; Stan, C.; Vişan, G.T.; Ganciu, M.; Filinov, V.; Lapitsky, D.; Deputatova, L.; Syrovatka, R. Multipole electrodynamic ion trap geometries for microparticle confinement under standard ambient temperature and pressure conditions. J. Appl. Phys. 2016, 119, 114303. [CrossRef] 